\title{
National Program on High Field Accelerator Magnet R\&D
}

\author{
White Paper submitted to the 2014 HEPAP Accelerator R\&D Subpanel \\ G. Apollinari, L. Cooley, A.V. Zlobin (FNAL), S. Caspi, S. Gourlay, S. Prestemon (LBNL), \\ D. Larbalestier (NHMFL), R. Gupta, P. Wanderer (BNL)
}

September 26, 2014

\section{Executive summary}

A National High-Field Magnet (HFM) Program is proposed as a thrust of the updated DOEHEP General Accelerator R\&D Program. The program responds to Recommendation 24 of the 2014 Particle Physics Project Prioritization Panel (P5) Report. The program identifies 4 central goals:

1. Develop accelerator magnets at the limit of $\mathrm{Nb}_{3} \mathrm{Sn}$ capabilities.

2. Investigate accelerator magnet designs with Low Temperature Superconductor (LTS) and High Temperature Superconductor (HTS) coils for fields beyond the capability of $\mathrm{Nb}_{3} \mathrm{Sn}$.

3. Drive high-field conductor development, including $\mathrm{Nb}_{3} \mathrm{Sn}$ and HTS materials for high-field accelerator magnets.

4. Address fundamental aspects of magnet design, technology and performance that could lead to substantial reductions of magnet cost.

The Program addresses these goals in two phases over 6 years. In Phase 1 (FY15-FY17) the Program will focus on the development and test of a small-aperture high-field $(\sim 16 \mathrm{~T}) \mathrm{Nb}_{3} \mathrm{Sn}$ dipole demonstrator for the Future Circular Collider (FCC) studies, development of high-current $(\sim 10 \mathrm{kA})$ HTS cables and small insert coils using these cables, and accelerator magnet design studies to identify the possibilities of magnet cost reduction. Phase 2 (FY18-FY20) envisions a long-term transition to accelerator quality magnets relevant for $100 \mathrm{TeV}$ scale $p p$ collider, with integrated management of stress, grading of conductors, and cost savings resulting from design, technology and performance optimization. Following P5 Recommendation 23, the Program relies upon a collaborative effort of U.S. National Labs, industry and universities that takes maximum advantage of existing infrastructure and expertize at the participating institutions.

\section{Introduction}

The 2014 Particle Physics Project Prioritization Panel (P5) provided an updated strategic plan for U.S. HEP to realize a twenty-year global vision for the field [1]. This plan describes a prioritized list of projects and their timelines that are required to address the science drivers identified by P5. According to the P5 report, "A very high-energy proton-proton collider is the most powerful future tool for direct discovery of new particles and interactions under any scenario of physics results that can be acquired in the P5 time window... The U.S. is the world leader in R\&D on high-field superconducting magnet technology, which will be a critical enabling technology for such a collider." The report authorizes:

Recommendation 24: Participate in global conceptual design studies and critical path $R \& D$ for future very high-energy proton-proton colliders. Continue to play a leadership role in 
superconducting magnet technology focused on the dual goals of increasing performance and decreasing costs.

U.S. National Laboratories, industries and the university programs, supported by DOE-OHEP, provided critical contributions to the advance of superconducting accelerator magnet technologies during the past four decades. The impressive achievements of U.S. accelerator magnet R\&D include world records in field strength and field gradient of magnets, successful technology industrialization and application in practical accelerators, and growth of the world's biggest superconductor industry in the USA. Superconducting $\mathrm{Nb}-\mathrm{Ti}$ magnets are the essential components of the Tevatron, HERA, RHIC, and most recently the LHC. The $\mathrm{Nb}_{3} \mathrm{Sn}$ magnet technology being successfully developed in the U.S. by the LARP collaboration provides the strong foundation for the high-luminosity LHC upgrade (HL-LHC) project that is now entering its construction phase.

Building on this leadership history in accelerator magnet R\&D, we believe that we should refocus our program towards cost-effective and transformational technologies essential for the future success of HEP. Frontier accelerator magnets at fields of $\sim 16 \mathrm{~T}$ based on $\mathrm{Nb}_{3} \mathrm{Sn}$ and $\sim 20 \mathrm{~T}$ based on HTS in combination with $\mathrm{Nb}_{3} \mathrm{Sn}$ are a central choice of the Future Circular Collider (FCC) study centered at CERN [2] and at the CEPC/SppC study in China [3]. Since this choice is in large measure based on experience in the U.S. high field magnet program, we propose a focused U.S. superconducting accelerator magnet and conductor $R \& D$ program on a scale appropriate to limited budgets, while still educating the next generation of magnet scientists and engineers. A strengthened National HFM R\&D Program will also play a critical role in risk mitigation of LARP and HL-LHC project by providing resources in the form of facilities and skilled personnel.

This White Paper describes a National HFM R\&D Program, a key thrust of the updated DOEOHEP General Accelerator R\&D (GARD) Program (described in the separate White Paper [2]), which responds to the P5 recommendations, has the proper balance of R\&D under the different budget scenarios envisioned by P5, and ensures that the U.S. maintains world leadership in highfield SC accelerator magnet technology. The Program is a collaborative effort of U.S. National Labs, industry and universities that takes advantage of existing infrastructure and strengths, consistent with the P5 Recommendation 23.

Recommendation 23: Support the discipline of accelerator science through advanced accelerator facilities and through funding for university programs. Strengthen national laboratory-university $R \& D$ partnerships, leveraging their diverse expertise and facilities.

\section{The Envisioned National Program}

The Program aims at the following goals:

Goal 1: Develop accelerator magnets at the limit of $N b_{3}$ Sn capabilities. This is presently believed to be approximately $16 \mathrm{~T}$.

Goal 2: Explore LTS accelerator magnets with HTS inserts for fields beyond the $\mathrm{Nb}_{3} \mathrm{Sn}$ capabilities. The present target is $20 \mathrm{~T}$ or above.

Goal 3: Drive high-field conductor development, both $\mathrm{Nb}_{3}$ Sn and HTS materials, for accelerator magnets.

Goal 4: Address fundamental aspects of magnet design, technology and performance that could lead to substantial reduction of magnet cost. 
The proposed Program consists of two phases, Phase 1 focused on providing input for the FCC CDR in 2018, and Phase 2 setting the foundation for the development of high-field accelerator magnets over the longer term.

\section{i) Phase 1 (FY15-FY17)}

1. Development and test of a small-aperture $16 \mathrm{~T} \mathrm{Nb_{3 }}$ Sn dipole based on traditional cos-theta design as a baseline demonstrator of an FCC dipole. This magnet will also be used to test the first small HTS dipole inserts.

2. Development and test of small-aperture $16 \mathrm{TN} \mathrm{N}_{3}$ Sn dipole based on common coil design. This magnet will also be used for racetrack HTS insert coils.

3. Development and test of a multi-layer $\mathrm{Nb}_{3} \mathrm{Sn}$ Canted Cosine Theta $(C C T)$ dipole reaching a field close to $16 \mathrm{~T}$ and early demonstration of the suitability of the CCT design for HTS inserts.

4. Magnet design studies and cost optimization. Design concepts of twin-aperture 15-16 T dipoles based on large cables, graded coils, and cold and warm iron yokes will be developed and compared with the goal of reducing magnet cost (\$/Tesla) by a factor of 3-4 with respect to the present LHC main dipole.

5. Development of $\mathrm{Nb}_{3}$ Sn and HTS (Bi-2212 and REBCO) strands and high-current cables. The work on the $\mathrm{Nb}_{3} \mathrm{Sn}$ will focus on increasing high-field conductor current density in cable forms in collaboration with the Conductor Development Program (CDP) and Oxford Superconducting Technology (OST). The work on Bi-2212 and ReBCO strands and cable will concentrate on optimized strand development and demonstration of $10 \mathrm{kA}$ class cables for HTS dipole inserts. The level and scope of work on ReBCO tapes and cables will be coordinated with similar effort by EuCARD2 and CERN.

In Phase 1 the Program combines a cost-effective high-risk/high-payoff potential with usage, wherever possible, of existing and proven platforms instead of "design-from-scratch optimization" that would be possible only in a more optimally and generously funded R\&D program. The envisaged outcomes expected over the initial 3-year phase I will maintain U.S. leadership in highfield accelerator magnet technology. This timescale coincides with the CERN's FCC study. This will naturally set the stage for a longer and potentially internationally combined effort.

ii) Phase 2 (FY18-FY20)

1. Development and optimization of a small-aperture $16 \mathrm{~T} \mathrm{~N} \mathrm{~N}_{3}$ Sn dipole. Within the first three years of the Program, significant progress is anticipated in defining the magnet requirements of a future hadron collider. Depending on the outcome of the Phase 1 and CERN effort to build and test the 16 T block-type dipole (FRESCA-2) by 2018, there will likely be a design downselect for more focused magnet development.

2. Development and test of a large-aperture $\sim 15 \mathrm{~T} \mathrm{~N} b_{3}$ Sn dipole. The large-aperture $\mathrm{Nb}_{3} \mathrm{Sn}$ dipole will provide a $15 \mathrm{~T}$ background field for a 5-10 T HTS insert aimed at generating a 20 $\mathrm{T}$ magnet. This development will also demonstrate the feasibility of large-aperture high-field magnets required for FCC interaction regions.

3. Development and study of $\mathrm{Nb}_{3}$ Sn and HTS strands and high-current cables in support of magnet program. Depending on the outcome of the ReBCO and Bi-2212 cable and insert coil 
R\&D, and taking into account the results of EuCARD2 program, there will likely be a HTS conductor down-select for HTS inserts.

4. Magnet design studies and cost optimization. Design studies to optimize the conceptual design of dipole magnet will continue, focusing on cost analysis of accelerator magnet systems and the contributions of magnets to the total accelerator cost, as well as opportunities for substantial cost reduction, as required by the P5 report.

\section{Relationship to Other Programs}

The envisioned program requires close coordination with other R\&D programs, and assumes the continued activity in other programs.

The U.S. Conductor Development Program (CDP) will be relied upon to support appropriate modifications and improvements of the $\mathrm{Nb}_{3} \mathrm{Sn} \mathrm{RRP}^{\circledR}$ conductor, investigation of alternative $\mathrm{Nb}_{3} \mathrm{Sn}$ conductor technologies, as well as to support the continued development of HTS conductors, conductor cost analysis and reduction.

Continued interaction with EUCARD2 and CERN will be an important ongoing activity. At present, EUCARD2 has ReBCO as its primary option, partly due to the strong U.S. commitment to develop Bi-2212 technology. CERN effort to build and test the $16 \mathrm{~T} \mathrm{Nb}_{3} \mathrm{Sn}$ block-type dipole (FRESCA-2) by 2018 will provide information on this alternative magnet design which is not supported in Phase 1 of the described National Program due to limited funding in budget scenarios $\mathrm{A}$ and $\mathrm{B}$.

In addition, activities in China, Korea, and Japan need to be monitored. For example, Kyoto University will host the next discussion (November 2014) of high-field conductor and magnet development for accelerators, building on the recent discussions in Hamburg (May 2014) and Geneva (February 2014).

\section{Program funding}

The envisioned program requires an additional $\sim 5 \mathrm{M} \$ /$ year compared to FY14 under budget scenario A and B. Due to downward trends in recent budgets, the programs at the participating institutions no longer have flexibility to respond to budget reductions, which might occur under scenario A, which was pointed out by the P5 report. Budget scenario A could result in the loss of key deliverables for the FCC study, the loss of conductor development, or the loss of technology pathways around inherent limitations of high-field cosine-theta dipoles. Any of these losses could have lasting negative impact on the science drivers identified by P5. Under budget scenario C, development of high-field magnets and high-field conductors will accelerate, resulting in the appearance of FCC-relevant technology many years sooner.

\section{References}

[1]'Building for Discovery: Strategic Plan for U.S. Particle Physics in the Global Context," P5 Report, http://science.energy.gov/ /media/hep/hepap/pdf/May\%202014/FINAL_P5_Report_053014.pdf

[2] "National General Accelerator R\&D Recommendations," Fermilab General Accelerator R\&D Priorities Working Group, September 19, 2014.

[3] Future Circular Collider Study Kickoff Meeting, Geneva, Feb. 12-14, 2014, http://indico.cern.ch/event/282344/timetable/\#20140212

[4] CEPC/SppC study in China, http://indico.cern.ch/event/282344/session/1/contribution/65/material/slides/1.pdf 